$\begin{array}{cl}\begin{array}{c}\text { Revue } \\ \text { de } / \text { histoire } \\ \text { des religions }\end{array} & \text { Revue de l'histoire des religions } \\ & \begin{array}{l}1 \mid 2013 \\ \text { Varia }\end{array}\end{array}$

\title{
Le singe miroir de l'homme ? Enjeux d'une confrontation en Grèce ancienne
}

The monkey as a mirror for man? The issues surrounding a confrontation in ancient Greece

\section{Karin Mackowiak}

\section{(2) OpenEdition}

\section{Journals}

Édition électronique

URL : http://journals.openedition.org/rhr/8058

DOI : $10.4000 /$ rhr.8058

ISSN : 2105-2573

Éditeur

Armand Colin

Édition imprimée

Date de publication : 1 mars 2013

Pagination : 5-36

ISBN : 978-2-200-92863-6

ISSN : 0035-1423

\section{Référence électronique}

Karin Mackowiak, «Le singe miroir de l'homme ? Enjeux d'une confrontation en Grèce ancienne », Revue de l'histoire des religions [En ligne], 1 | 2013, mis en ligne le 01 mars 2016, consulté le 19 avril 2019. URL : http://journals.openedition.org/rhr/8058 ; DOI : 10.4000/rhr.8058 


\section{Le singe miroir de l'homme? Enjeux d'une confrontation en Grèce ancienne}

La figure du singe en Grèce ancienne interroge autant l'historien de l'imaginaire, capté par une créature dont l'analyse iconographique reste embryonnaire, que l'anthropologue des religions, tenté d'y voir une expression emblématique de conceptions ontologiques et religieuses grecques. Si l'introduction de l'animal en Méditerranée et certaines de ses représentations figuratives doivent beaucoup à l'Orient, son univers, empreint des mystères des représentations de passage, doit d'abord à la Grèce $d u I^{e r}$ millénaire av. J-C ses contours particuliers. Le singe, miroir de l'homme, présentait sans doute un enjeu théologique que cette étude introductive, pensée en contrepoint avec les débats philosophiques du darwinisme, choisit d'éclairer.

\section{The monkey as a mirror for man? The issues surrounding a confrontation in ancient Greece}

The figure of the monkey in ancient Greece appeals both to the historian of the imagination, who is captivated by this creature whose iconographic study remains embryonic, and to the religious anthropologist, who is tempted to see in it an emblematic expression of Greek ontological and religious concepts. While the animal's introduction into the Mediterranean region, like some of its figurative representations, owes much to the East, the imagery surrounding monkeys, imbued with the mysteries of representations of passage, owes its particular contours to Greece in the first millennium BC. The monkey, a mirror for mankind, certainly presented a theological issue and this introductory study (conceived of in juxtaposition to philosophical debates on Darwinism) will attempt to shed light upon it. 
La relation de l'homme au singe est imprégnée du mystère et de la fascination qui nous renvoient à nos origines et à notre nature. Quel intérêt exactement le singe présente-t-il dans la religion grecque antique?

Présente dans les sanctuaires de la Grèce ancienne, l'image du singe peut être perçue par l'historien comme une curiosité méconnue à la valeur, en fait, anthropologique et universelle. Ici dans le polythéisme, là dans le monothéisme, le singe traverse les problématiques religieuses où il fait question. Les émois avivés au XIX ${ }^{e}$ siècle par Darwin, auteur d'une insertion sacrilège du singe entre l'homme et Dieu, viennent souligner la permanence d'une confrontation qui ne s'est jamais tue: le singe est-il le «miroir de l'homme»? À cette question répondent les pensées philosophiques et théologiques dont chaque civilisation se fait l'architecte. Cette confrontation entre l'homme et l'animal cache tout un édifice religieux et spéculatif, inséparable du rapport entre l'homme et le divin. Y avait-il un enjeu théologique du singe dans la culture grecque antique?

Ces questions sont d'autant plus légitimes à poser qu'elles amorcent un champ d'investigation nouveau à plusieurs titres. Jusqu'à présent, la figure du singe en Grèce n'a fait l'objet d'études approfondies qu'à partir des textes - dans les fables et le théâtre'. Pourtant l'animal intervient également dans l'iconographie et ce type de documentation, relativement négligé, mérite d'être considéré de plus près. Il reste à découvrir le cheminement temporel et culturel qui a mené les Hellènes à voir en cet animal une véritable expression symbolique d'altérité. En concordance avec certaines recherches sur l'animalité (progressivement citées infra), il apparaît d'abord que le singe peut contribuer à la définition du genre humain et à sa place dans le cosmos. Force est de constater, ensuite, des

1. Paul Demont, «Aristophane, le citoyen tranquille et les singeries » in: Pascal Thiercy et Michel Menu (éd.), Aristophane: la langue, la scène, la cité. Actes du colloque de Toulouse, (17-19 mars 1994), Bari, 1997, p. 457-479; Carlos G. Gual,

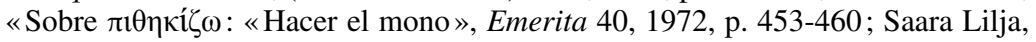
«The Ape in Ancient Comedy», Arctos 14, 1980, p. 31-38; Mario Vegetti, «L'animale ridicolo» in: Mario Vegetti (éd.), Tra Edipo e Euclide. Forme del sapere antico, Milan, 1983, p. 59-70. 
points communs saisissants entre la vision grecque de l'animal et la nôtre, ce qui nous conduit à évaluer cette créature dans le cadre d'une démarche également comparatiste, fût-elle modeste: l'Orient antique et la pensée chrétienne nous aident à rehausser, en creux, les contours religieux aujourd'hui évanouis du «pithecos » grec, animal emblématique. Posons quelques jalons d'histoire et d'anthropologie religieuses sur des représentations imaginaires dont la richesse et la complexité demeurent insoupçonnées.

\section{SOURCES ET RESSEMBLANCES}

Rapprocher le singe et l'homme paraîtrait sans risque aucun s'il n'était le doute qui s'empare du chercheur aux prises avec des catégories presque trop familières: mettre en valeur les ressemblances entre l'homme et le primate ne risque-t-il pas de subordonner la vision grecque de l'animal à la nôtre? Il ne nous échappe jamais, dans la contemplation de cette créature, combien elle apparaît comme la copie de l'homme, tantôt amusante tantôt pitoyable, mimésis dévaluée dans tous les cas. En 1875, l'abbé Maudoit exploite opportunément le singe dans ses objectifs pédagogiques catéchistes, et la sentence est sans appel: «Qu'est-ce que l'homme? Savez-vous, mes enfants, comment de prétendus savants répondent à cette question? L'homme, disent-ils, est un singe perfectionné. Un singe, vous savez, ce vilain petit animal que vous avez peut-être vu sur le champ de foire. La réponse est-elle à votre goût? Et vraiment est-ce la peine d'avoir tant d'esprit pour dire qu'on n'est qu'une bête ? $»^{2}$.

L'homme grec de l'antiquité n'avait certes pas les mêmes opportunités d'observation: si dès avant l'époque hellénistique diverses espèces de singes circulaient sur les routes commerciales de la Méditerranée, les cercopithèques et autres cynocéphales n'y avaient certainement pas la même présence que dans notre

2. Régis Ladous, «Adam, le singe et le prêtre. La question des origines et de l'évolution biologique de l'homme dans les catéchismes français (1850-1950) in: Christianisme et Science, Paris, 1989 (Association française d'histoire religieuse contemporaine), p. 101. Voir de même les remarques de Harry W. Paul, The Edge of Contingency. French Catholic Reaction to Scientific Change from Darwin to Duhem, Gainesville, Floride, 1979, p. 48. 
civilisation où les parcs zoologiques et toutes sortes de supports médiatiques plantent ces créatures dans un paysage familier. Et pourtant, l'animal laisse de nombreuses traces dans la culture antique.

Les textes grecs s'en font les meilleurs échos. Dans Ésope, le singe incarne des tares précises et récurrentes, reprises ensuite dans la comédie classique. Le primate est par excellence l'incarnation du ridicule et de la laideur psychologique de l'homme: il est vaniteux, menteur, stupide et vil ${ }^{3}$. Mais la familiarité des Grecs avec le singe s'exprime d'une manière autrement plus intéressante lorsque l'on observe le corpus méconnu des images. Si les vases archaïques et classiques ont attiré l'attention des spécialistes ${ }^{4}$, les figurations simiesques sur la coroplastie sont plus riches encore, tant du point de vue de la quantité des représentations que de leur amplitude chronologique.

À l'époque archaïque grecque, la figuration du primate est récurrente de la Méditerranée orientale à la Grèce continentale et égéenne ${ }^{5}$ où sont élaborées des images pour le moins originales.

3. Ésope, Fables 38, 39, 145, 304, 306 et 307. Le fabuliste a développé des traditions dont le récit fragmentaire du singe et du renard d'Archiloque (fragments 185-187 West) est l'écho le plus ancien conservé.

4. Herman A.G. Brijder, «Apish Performances in the 6th Cent. BC», in J. Christiansen et T. Melander (éd.): Proceedings of the $3^{\text {rd }}$ Symposium on Ancient Greek and Related Pottery. Copenhagen August 31 - September 4, 1987, Copenhague, 1988, p. 62-70; Jaap M. Hemelrijk, Caeretan Hydriae, Mayence, 1984 (Kerameus 5, J. Boardman et H. Cahn dir.), pl. 38, 52 et 81; François Lissarague, «L'homme, le singe et le satyre», in: Barbara Cassin, JeanLouis Labarrière et Gilbert Romeyer Dherbey (éd.), L'animal dans l'antiquité, Paris, 1997, p. 455-472; Martin Robertson, «A muffled dancer and others», in Alexander Cambitoglou (éd.), Studies in honour of Arthur Dale Trendall, Sydney, 1979, p. 129-134; Dietrich Von Bothmer, The Amasis Painter and His World. Vase-Painting in sixth-century B.C., Athènes, Malibu, New York, 1985, p. 219. Une synthèse iconographique à visée exhaustive, mais vieillie, demeure celle de William C. Mac Dermott, The Ape in Antiquity, Baltimore, 1938.

5. À Rhodes: Reynold A. Higgins, Catalogue of the Terracottas in the Department of Greek and Roman Antiquities. British Museum, Londres, 1954, $\mathrm{n}^{\circ}$ 53/93/ 105 et 106; Franz Winter, Die Typen der figürlichen Terrakotten, I, Berlin, Stuttgart, 1903, n 224, 1. À Samos: Nicholas C. Stampolidis (éd.), Ploes, Sea Routes... From Sidon to Huelva. Interconnections in the Mediterranean, 16th-6th c. $B C$, Athènes (Museum of Cycladic Art), 2003, $\mathrm{n}^{\circ} 338$. À Mélos: F. Winter, op. cit. 223, 2a. À Théra: Hans Dragendorff, Theräische Gräber, II, Berlin, 1903, p. 124, fig. 70. À Naukratis: M. J. Maximova, Les vases plastiques dans l'antiquité (époque archaïque), Paris, 1927, p. 115-116. À Corinthe: R. A. Higgins, op. cit., $\mathrm{n}^{\circ}$ 966; F. Winter, op. cit., ${ }^{\circ}$ 222, 5. En Béotie: R. A. Higgins, op. cit., n 774/796. À Mégare: F. Lissarague, op. cit. (note 4), fig. 15. À Argos: Charles Waldstein, The 
Il suffit de se pencher sur des figurines de bronze du viII ${ }^{\mathrm{e}}$ siècle av. J.-C. (illustration 1). Ces énigmatiques objets véhiculent un imaginaire différent de celui du $\mathrm{II}^{\mathrm{e}}$ millénaire oriental: des sortes d'hommes-singes semblent être représentées ${ }^{6}$. Le caractère mixte de ces formes est d'autant plus difficile à nier qu'on en trouve des parallèles dans la littérature. Au II $^{\mathrm{e}}$ s. av. J.-C., le géographe Agatharchidès de Cnide rapporte qu'il existerait aux confins de la terre des singes au corps humain ${ }^{7}$. Une vision archaïque de l'animal paraît avoir perduré durant de nombreux siècles, perception aucunement scientifique mais néanmoins pointue. Le pithecos est proche de l'homme mais en même temps mixte et inférieur. Il reste pourtant moins jaugé en termes moraux qu'en termes de sauvagerie: Agatharchidès qualifie les cynocéphales d' «agrion», «vivant à l'état sauvage», suivant un qualificatif qui peut également concerner des hommes ${ }^{8}$. Il y a donc dans cette perception une forme de similitude avec le regard de l'homme moderne: la conscience d'une proximité entre l'être humain et le primate fascine dans les deux cultures, ou inquiète; et, pareillement, l'attrait pour le primate confine à la répugnance ou au déclassement. Mais alors que la régression de l'homme à l'animal est inadmissible dans le christianisme, cette dernière fait partie d'un plan du monde dans

Argive Heraeum, II, Boston, New York, 1902, $\mathrm{n}^{\circ}$ 264. Exportations ioniennes et corinthiennes en Grande Grèce: M. I. Maximova, op. cit., $\mathrm{n}^{\circ}$ 57/155. Ces mentions sont indicatives de la variété géographique, mais le nombre d'objets découverts est bien plus important.

6. Susan Langdon, «From Monkey to Man: the Evolution of a Geometric Sculptural Type», American Journal of Archaeology, 94, 1990, p. 407-424.

7. De Mari Erythraeo, 74, où, plus précisément, Agatharchidès considère les singes cynocéphales comme des hommes déformés : au corps humain à l'exception de la tête, ce genre de créature possède un type de langage, lâchant des sons qui ressemblent à des grognements. Ce genre de créature ne peut absolument pas être apprivoisé et montre un air menaçant. Cela les éloigne des espèces totalement rejetées du côté animal de par leur caractère domesticable (73) ou de par leur physique sans ambivalence (75), tout ce que les cynocéphales ne sont pas (= Diodore de Sicile, Bibliothèque historique, III, 35, 5).

8. Sont désignés comme tels des hommes identifiés comme partiellement animaux: cette vision est explicite dans les Histoires d'Hérodote où les agrioi de Libye sont conçus comme des êtres hybrides du type des cynocéphaloi et des acéphaloi mentionnés dans la même phrase, en IV, 191: voir Stefania De Vido, «Belve, scimmie, uomini nella Libia erodotea», in: Ettore Cingano, Antonella Ghersetti et Lucio Milano (dir.), Animali tra zoologia, mito e letteratura nella cultura classica e orientale. Atti del Convegno di Venezia, 22-23 maggio 2002, Padoue, 2005, p. 197-198. 
la pensée grecque, ne serait-ce qu' au sens géographique du terme ${ }^{9}$ : les formes mixtes sont constitutives du cosmos et elles valent comme règles dans une définition graduée de la civilisation et de l'humanité. Le rapprochement, possible, de l'homme avec l'animal intègre une structure de l'imaginaire normée. De ce fait, le mixte n'a rien que de très banal dans la pensée grecque, apte à abolir le fossé infranchissable entre singe et homme qui peut prévaloir ailleurs.

\section{DES DIFFÉRENCES : APPROXIMATION PHYSIQUE DU SINGE EN GRÈCE}

Ce paysage imaginaire posé, qu'en est-il donc du religieux ? Deux figurines grecques ont le mérite d'être particulièrement explicites quant à la présence du singe dans un contexte imaginaire religieux : une figurine de Mélos et une autre de Sélinonte (illustrations 2 et 3). La première représente un petit homme grassouillet; la seconde, découverte dans le sanctuaire de la Malophoros, figure vraisemblablement la déesse, image de la Déméter locale ${ }^{10}$. À chaque fois un petit singe est juché sur l'épaule du personnage principal. Cette iconographie du VI ${ }^{\mathrm{e}} \mathrm{s}$. av. J.-C. s' articule dans un cadre cultuel ostensible - au moins en ce qui concerne la Malophoros.

De quelles croyances peut-il s'agir? C'est à la même époque, le $\mathrm{VI}^{\mathrm{e}} \mathrm{s}$. av. J.-C., que le singe devient populaire dans la littérature et se pare des attributs drolatiques qui le caractériseront au siècle suivant. Par contre, les textes ont perdu toute trace de cet univers religieux : ce dernier ne survit donc qu'au travers d'images muettes qu'il faut essayer de faire parler. Outre le caractère votif de certaines d'entre elles, la mise en forme des figurines laisse encore transparaître un dénominateur commun, capable d'expliquer la présence du pithecos: dans l'un comme dans l'autre cas, ces images plaident pour leur insertion dans l'univers féminin. En effet, l'hypothèse de croyances courotrophes le dispute à celle invoquant des pratiques sexuelles patronnées par une déesse

9. Sur la classique vision ethnocentrique du monde chez Hérodote et ses conséquences pour ce qui est du singe, voir S. De Vido, op. cit. (note 8) p. 195-204.

10. Respectivement: R. A. Higgins, op. cit. (note 5) n ${ }^{\circ} 93$; Ettore Gàbrici, Il santuario della Malophoros a Selinunte, Milan, 1927 (Monumenti Antichi pubblicati per cura della R. Accademia Nazionale dei Lincei, XXXII), pl. XL, 2. 
comme Cybèle ${ }^{11}$. Le singe, sur ces images, ne s'articulerait-il donc pas à un imaginaire du passage?

Rien n'empêche de considérer la créature comme une sorte de corps incomplet, en gestation, proche de ce que la littérature ethnologique nous en confie: une forme mixte. L'idée de transformation du corps, mise sous la tutelle d'une divinité courotrophe ou comprise comme changement graduellement imperceptible, n'explique-t-elle pas la présence du singe ? Ce dernier n'aurait-il pas la faculté d'expliciter, par ses contours corporels imprécis, un monde de l'entre-deux, celui entre enfance et âge adulte par exemple? N'appuie-t-il pas l'idée d'un état intermédiaire que les Grecs auraient, dans le cadre des croyances méliennes comme sélinontaines, intégré dans le sacré?

Bien au-delà des leitmotivs littéraires friands de singes ridicules et fondateurs (pour partie) du point de vue moderne, la culture grecque a sans aucun doute su réinventer l'animal dans l'univers religieux. Elle lui a conféré une place tout à fait remarquable lorsqu'on sait combien le corps beau s'est imposé dans cette civilisation. Mais l'affirmation d'une mixité entre corps humain et corps simiesque ne semble plus poser problème dès lors qu'entre en jeu une définition de l'humanité évaluée selon les termes d'une graduation. Sauf que cette dernière n'est plus d'ordre géographique mais religieux, concernant toujours la place de l'homme dans le cosmos. Se trouvet-on en présence d'une définition de l'humanité qui s'exprime en termes de seuil à franchir, et ce par le biais d'une créature d'altérité ? Se trouve-t-on en présence de croyances religieuses normées dans des sanctuaires où l'idée de seuil passe nécessairement par un stade de régression?

Une observation s'impose à propos de la figurine de Mélos. L'hypothèse d'une connexion entre le nain et des pratiques sexuelles masculines apparaitt particulièrement convaincante au regard de la comparaison établie par Ursula Mandel entre la posture de ce petit sujet et celle d'un jeune homme figuré sur une coupe fragmentaire

11. Respectivement Theodora Hadzisteliou-Price, Kourotrophos. Cults and Representations of the Greek Nursing Deities, Leyde, 1978, p. 76 et p. 181sq., et Ursula Mandel, «Ägyptische Schemata in ostgriechischer Aneignung: Figürliche Salbgefäße und Terrakotten archaischer Zeit», in: Herbert Beck, Peter C. Bol et Maraike Bückling (dir.), Ägypten, Griechenland, Rom. Abwehr und Berührung. Städelsches Kunstinstitut und Städtische Galerie, 26. November 2005-26. Februar 2006, Frankfort, 2005, p. 47-70. 
laconienne (illustration 4) ${ }^{12}$ : ce sont plus précisément des croyances de confréries archaïques qui s'esquisseraient en arrière-plan, mettant, le temps d'une initiation, l'individu aux prises avec son propre statut. Ces représentations imaginaires ne prennent sens que dans le cadre d'un passage par un stade régressif, liminal. Or, cette coupe laconienne figure, par un détail qui n'a jamais été noté, un singe: en effet, en bas du registre supérieur figurant le cômos, une main poilue retenue par une laisse se laisse apercevoir. La surprise s'estompe alors en constatant la représentation d'un tel animal sur le nain de Mélos; sa présence est sans doute due à sa signification symbolique: dans cette archaïque vision du monde, les Grecs semblent avoir perçu le singe comme un petit corps atrophié qui, au plan religieux, pourrait bien énoncer un phénomène en devenir, une forme de temporalisation sacralisée ${ }^{13}$.

La régression est encore palpable sur un autre document, un vase connu: la belle coupe de la Villa Giulia ${ }^{14}$. Celle-ci figure des singes banqueteurs, improbables acrobates en pleine prouesse absurde qui disent la régression orgiaque du banquet - dionysiaque donc. En lieu et place d'un contexte religieux, c'est l'atmosphère aristophanesque qui ressort, l'idée du singe maladroit et glouton. Mais est-ce un hasard si le peintre a choisi, pour dire la régression dionysiaque, des singes? Jusqu'à ce jour, les chercheurs se sont demandés s'il s'agissait de singes ou d'hommes grimés ${ }^{15}$. Mais plutôt que de considérer le réalisme de ce genre de scène, ne faut-il pas y voir avant toute chose une dimension métaphorique, symbolique, celle propre au singe? Auquel cas, cette image se ferait la trace résiduelle de son rôle dans la sphère religieuse.

Le ludique occupe sans aucun doute une place centrale sur cette image. Mais de la céramographie à la coroplastie on note des cohérences d'un autre ordre dont d'autres figurations du $\mathrm{V}^{\mathrm{e}} \mathrm{s}$. av. J.-C. se font les témoins. Une image énigmatique, inédite jusqu'à une date récente, conservée à l'Ashmolean Museum, mérite d'être

12. U. Mandel, ibid., p. 55sq.

13. Cette hypothèse sera développée dans le cadre d'un travail ultérieur mené dans le cadre de l'opération «Temps, dieux et temporalité» de l'axe 2 de l'Institut des Sciences et Techniques de l'Antiquité (Besançon).

14. Par exemple dans F. Lissarague, op. cit. (note 4), fig. 9.

15. La question est posée en ces termes depuis l'analyse de H.A.G. Brijder, op. cit. (note 4). 
regardée de plus près (illustration 5): elle représente une créature debout, jambes écartées, dotée d'une queue et portant un objet dans les mains ${ }^{16}$. Cet objet présente de fortes similitudes avec d'autres: des figurines de satyres ou de grotesques représentées avec les mêmes attributs. L'une d'elle, inédite elle aussi et conservée au Musée National de Berlin nous donne une idée de ce type d'images qui semblent avoir été assez répandues à l'époque classique. L'illustration 6 nous montre un personnage au corps humain mais au visage nettement plus ambigu: la partie inférieure de ce dernier présente une excroissance mandibulaire qui évoque une protubérance simiesque (illustration 7). La tête est moins animale que la créature de l'Ashmolean mais n'est pas pour autant humaine: c'est encore la trace d'une remarquable fusion entre corps humain et animal ${ }^{17}$. Outre la période de production, un autre point commun unit ces sujets : ils proviennent en fait de la même aire géographique, la Béotie. La série parallèle des satyres et grotesques fut quant à elle découverte à Égine mais rien n'interdit d'y voir une connexion avec la Béotie voisine, vu la gémellité des motifs ${ }^{18}$. Ces images ont fait l'objet d'une production standardisée qui a circulé entre la Grèce centrale et l'Égée occidentale.

Une finalité probable de ces petits objets est leur fonction d'exvoto. Bon nombre de ces personnages tiennent dans la main un animal, évoquant la gestuelle de bergers ou de donateurs, dans tous les cas de fidèles ${ }^{19}$. L'identité de l'orant peut surprendre puisque le singe se substitue parfois à l'homme. Mais, dans cette série d'argile modelée, il y a finalement cohérence: singes et grotesques sont uniformément dotés d'une grosse tête et d'une gestuelle similaire: corporellement ils sont mis sur le même plan et peuvent être interchangeables. À constater la provenance d'un certain nombre de ces figurines, une autre cohérence interpelle: leur rapport avec

16. Karin Mackowiak, «Singe comique? Remarques sur une figurine en terre cuite de Thèbes (Béotie)», Gerión 28 (1), 2010, p. 57-73.

17. À comparer avec Niels Breitenstein, Catalogue of Terracottas: Cypriote, Greek, Etrusco-Italian, and Roman. Danish National Museum, Department of Oriental and Classical Antiquities, Copenhague, 1941, $\mathrm{n}^{\circ} 318$.

18. N. Breitenstein, ibid., $\mathrm{n}^{\circ} 317$ à 321. En ce qui concerne les petits objets éginètes, j'ai prévu une publication spécifique à leur sujet dans Ktèma (à paraître: «Des usages du singe en Béotie: l'animalité entre discours et fonction»).

19. Folkert T. Van Straten, Hiera kala. Images of animal Sacrifice in Archaic and Classical Greece, Leyde, New York, Cologne, 1995, p. 55sq. 
le Cabirion thébain. Ce sanctuaire est précisément connu pour avoir produit une céramique peuplée d'êtres contrefaits et macrocéphales parmi lesquels apparaît le bestiaire simiesque ${ }^{20}$. Une norme figurative locale paraît donc se dessiner: cette région de Grèce centrale a favorisé une iconographie susceptible de concerner de près les fidèles.

La figurine de l'Ashmolean fut acquise en 1934 à Thèbes. Bien sûr, elle ne s'insère pas de manière sûre dans un contexte spécifiquement cabirique mais elle rappelle étrangement la norme figurative propre à l'artisanat du sanctuaire thébain, friand d'une difformité à laquelle le singe se prête idéalement. On peut donc légitimement se demander si la figurine de l'Ashmolean Museum ne renvoie pas à la figure du dédicant, dans la continuité du théâtre où le pithecos apparaît comme le parasite du bômos. Mais pourquoi d'éventuels dédicants seraient-ils représentés comme des singes?

À l'échelle de la culture plus généralement grecque, cette hypothèse n'est en rien extravagante. On a évoqué la plausibilité d'«hommes-singes», dans l'imaginaire hellénique du viII ${ }^{\mathrm{e}} \mathrm{s}$. av. J.-C. jusqu'à la littérature hellénistique. Pendant ce laps de temps, les singes ambigus de la coroplastie classique ne constituent peut-être qu'une expression imaginaire parmi d'autres, une étape possiblement jalonnée par des croyances spécifiques visibles dès la fin $\mathrm{du} \mathrm{VI}^{\mathrm{e}}$ s. av. J.-C. Après le nain de Mélos et la Malophoros de Sélinonte (illustrations 2 et 3), connexions possibles du pithecos à des divinités féminines, vient le $\mathrm{v}^{\mathrm{e}} \mathrm{s}$. av. J.-C., lequel impose davantage l'univers dionysiaque, connu pour déchaîner le rire via tout un bestiaire - les satyres n'en sont que les plus connus. Or, dans la série des figurines de terre cuite béotiennes comme éginètes, les satyres ne manquent pas, dotés d'une corporéité exactement

20. Sur la céramique cabirique, voir Michèle Daumas, Cabiriaca: recherches sur l'iconographie du culte des Cabires, Paris, 1998 ou Jean-Marc Moret, «Circé tisseuse sur les vases du Cabirion», Revue archéologique, 2, 1991 (N.S.), p. 227266. Pour des figurines de grotesques, voir Karin Braun et Thea E. Haevernick, Bemalte Keramik und Glas aus dem Kabirenheiligtum bei Theben, IV, Berlin, 1981, qui abonde en êtres difformes, ou Bernhardt Schmaltz, Terrakotten aus dem Kabirenheiligtum bei Theben V. Menschenähnliche Figuren, Menschliche Figuren und Gerät, Berlin, 1974 pour la grotesque n ${ }^{\circ} 418$, découverte au nord du temple du sanctuaire. Pour le rapport des fouilles initiales: Peter Wolters, «Das Kabirenheiligtum bei Theben», Mitteilungen des Kaiserlich deutschen archaeologischen Instituts. Athenische Abteilung, 15, 1890, p. 363-364. 
similaire à leurs congénères simiesques et grotesques ${ }^{21}$. Du point de vue d'une hypothétique exploitation de la difformité, cela fait question: comme le satyre, le singe n'exhibe-t-il pas une liminalité de l'homme?

Il faut alors invoquer l'hypothèse, dans l'univers religieux grec, d'une véritable construction iconographique de la difformité qui remonterait à l'époque archaïque et qui, au fil du temps, aurait évolué et changé de sens en s'adaptant à des croyances diverses focalisées sur une conception commune de l'homme. Mais la modestie des objets considérés évoque aussi, il est vrai, une autre question qu'on ne peut éluder avant d'expérimenter plus loin notre raisonnement: celle de la médiocrité artisanale des images considérées. Animal extérieur au monde hellénique, quoique transitant de longue date par les routes du commerce égéen, le singe s'immisce dans l'art grec par le biais d'images bizarres où l'impression d'une grossière créativité vient forcément à notre esprit: l'espèce simiesque prête plus d'une fois le flanc au doute comme le montre par exemple l'illustration 8. Certes, la Béotie profonde, où ce genre de figurines a été produit par dizaines entre le premier et le dernier quart du $\mathrm{VI}^{\mathrm{e}} \mathrm{s} .{ }^{22}$, ne bénéficiait pas des acquis techniques d'artisanats autrement plus élaborés. L'Égypte millénaire est la première à venir à notre esprit, où les artisans travaillaient pour une aristocratie raffinée: ils reproduisaient un bestiaire simiesque où l'espèce zoologique demeurait clairement identifiable.

Mais ce constat ne satisfait pas entièrement l'historien anthropologue frappé par la récurrence d'une hybridité qui dépasse largement la Béotie: en coroplastie, le singe garde des contours corporels imprécis jusqu'à l'époque hellénistique, dans une mesure qui laisse place à l'intention délibérée ${ }^{23}$. À une période, donc, où l'animal n'a plus de secret pour les Grecs et où la production de

21. N. Breitenstein, op. cit. (note 17), nº 317 et 319 à 321 ou F. Winter, op. cit. (note 5), n 219, 5 et 219, 7 à 10.

22. Se reporter aux références bibliographiques citées supra note 5 .

23. Voir par exemple l'anatomie faciale pour le moins approximative de singes dans Simone Besques, Catalogue raisonné des figurines et reliefs en terre-cuite grecs, étrusques et romains. III. Époques hellénistique et romaine, Grèce et Asie mineure, Paris, 1972, $\mathrm{n}^{\circ}$ E/D 1277 ou E/D 2441. Peter Blome, «Affen im Antikenmuseum », in: Margot Schmidt et Ernst Berger (dir.), Kanon. Festschrift Ernst Berger zum 60. Geburtstag am 26. Februar 1988 gewidmet, Bâle, 1988 (Antike Kunst, Beiheft 15), p. 206-207, formule les mêmes remarques pour une statuette de singe en bronze, conservée au musée de Bâle (Inv. Me 10), datée 
figurines est sérielle (moulée), le pithecos semble bien continuer à fouetter l'imagination et à flotter avec conviction sur les plus improbables mirages - souvenons-nous de la description si sérieuse d'Agatharchidès: les cynocéphales ne sont autres que des hommes laids à tête de chien... tandis qu'une autre tradition prête des corps de singe à des hommes ${ }^{24}$.

$\mathrm{La}$ récurrence plaide pour une représentation corporelle volontairement floue de l'animal qui n'est pas à sous-estimer. C'est pourquoi l'historien des religions peut légitimement traquer derrière certaines formes, négligeables en apparence, la question d'une confrontation pluriséculaire de l' homme à l' animal. Ce jeu de miroir n'a jamais été, au fil du temps, question vaine dans les cultures. Ambigu, copie déformée de l'homme, le singe ne poserait-il pas chez les Grecs, et suivant un raisonnement tout à fait particulier, la question du rapport de l'homme à lui-même? Cet animal n'apparaîtrait-il pas en médiateur d'une question philosophique que l'homme grec a élaborée sur sa propre nature? Enfin, ce type de réflexion ne relève-t-il pas d'une forme de relativisme anthropocentrique forgé, si l'on remonte le temps, de la Grèce jusqu'à l'Orient antique? C'est dans les contrées orientales que la vision du singe révèle ses plus anciens contours anthropologiques, berceau d'une confrontation de l'homme à lui-même par le biais de l'animal... et par le biais du rire.

\section{SPÉCIFICITÉ DU I ${ }^{\mathrm{er}}$ MILLÉNAIRE GREC: \\ L'APPROXIMATION EST UN IMAGINAIRE}

Une enquête du côté de l'Orient du II ${ }^{\mathrm{e}}$ millénaire av. J.-C. - dont je ne peux exposer ici le détail - fait valoir deux caractéristiques majeures de l'iconographie simiesque. En premier lieu, le singe se fait déjà miroir de l'homme en motivant le rire. La facétie marque souvent la créature dont l'animalité est déjà réputée pour être toute

de la fin du $\mathrm{II}^{\mathrm{e}}$ ou du $\mathrm{I}^{\mathrm{er}}$ S. av. J.-C.: les traits simiesques sont flous mais pas méconnaissables, évoquant le type du nain.

24. Supra note 8. On ajoutera à ce dossier le cas de Thersite dont une scholie à l'Alexandra de Lycophron (1000) précise bien que son corps avait une forme

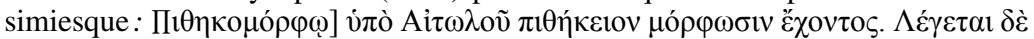

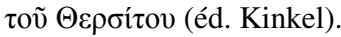


relative : placé en position de mimétisme, le singe pose la question de la frontière exacte de l'humanité, du Moyen Orient jusqu'en Égypte, berceau principal de la mise au point artistique du singe ridicule ${ }^{25}$. L'helléniste qui examine les figurines grecques du Ir millénaire av. J.-C. ne peut manquer de voir dans cet univers oriental les prémices d'un genre, d'un folklore que les Hellènes se sont empressés de reproduire $^{26}$. Mais à la différence de l'Égypte et du Moyen Orient où les figurations de singes respectent la physionomie animale, selon une fidèle observation de la nature, la culture grecque choisit de prendre une liberté autrement plus ample vis-à-vis du singe, plus exactement vis-à-vis de sa forme.

Pourtant, à en rester au plan des ressemblances, mêmes relatives, il convient de souligner une seconde caractéristique majeure, une sorte d'écho avant l'heure qui, faute d'un examen grec de la question, n'a pas encore été souligné: l'art oriental, et surtout égyptien, intégrait déjà le singe dans l'univers religieux. Il suffit de se remémorer les babouins assis en majesté ou saluant le lever du soleil, ainsi que leur connexion au dieu Thot $^{27}$. De ce point de vue, nos petites terres cuites acquièrent

25. Voir par exemple: l'homme au singe de Suse (= Agnès Spycket, Les figurines de Suse. 1. Les figurines humaines, $I V^{e}-I I^{e}$ millénaires av. J.-C., Paris, 1992 (Mémoires de la Délégation archéologique en Iran, tome LII), n 828); les ivoires de Nimrud (= Alan Houghton Brodrick, Animals in Archaeology, Londres, 1972, fig. 29 p. 57); les ostraca de Deir El Medineh (= Emma Brunner-Traut, Altägyptische Tiergeschichte und Fabel. Gestalt und Strahlkraft, Darmstadt, 1968, p. 2-3 ou Patrick F. Houlihan, Wit and Humour in Ancient Egypt, Londres, 2001, chapitre 3). Pour l'Égypte, les exemples abondent: il suffit de se reporter par exemple à : Jeanne Marie Thérèse Vandier d'Abbadie, Catalogue des Ostraca figurés de Deir El Médineh, Le Caire, 1937; Christiane Desroches-Noblecourt, Amours et fureurs de la Lointaine. Clés pour la compréhension de symboles égyptiens, Paris, 1995; Thierry Enel et François-Xavier Héry, Animaux du Nil, animaux de dieux, Aix-en-Provence, 1993, etc.

26. Par exemple pour les figurines qui caricaturent les savants: F. Lissarague, op. cit. (note 4), fig. 14 ou Don M. Bailey, «A caricature of Socrates», American Journal of Archaeology, 78, 1974, fig. 4.

27. Voir les nombreux bas-reliefs ou statues: les célèbres objets du Louvre (Inv. D 31 et E 11153), le babouin colossal d'Hermopolis et bien d'autres sculptures en quartzite, bronze, granodiorite sans oublier les manuscrits, du Nouvel Empire jusqu'à la Basse-époque: Anne Eggebrecht, L'Égypte ancienne: 3000 ans d'histoire et de civilisation au royaume des pharaons, Paris, 1997; T. Enel et F.-X. Héry, op. cit. (note 25); Friedhelm Hoffmann et Matthias Steinhart, Tiere vom Nil, Wiesbaden, 2001; Stephen Quirke et Jeffrey Spencer, Le livre de l'ancienne Égypte, Paris, 1995 ; Ewan H. Turner (dir.), Aménophis III. Le pharaonsoleil, Paris, Réunion des musées nationaux, 1993. On n'oubliera pas les ouvrages toujours utiles de Jean-François Champollion, Panthéon égyptien, collection des personnages mythologiques de l'Ancienne Égypte d'après les monuments, Paris, 
un intérêt certain: elles paraissent bien renvoyer à une appropriation grecque du pithecos dans une dimension religieuse, laquelle a pu varier suivant les lieux et les époques. Les Grecs n'auraient pu adopter docilement ni comprendre les images égyptiennes. Mais ils n'ont pas manqué de laisser au compte du singe ce rire spontané et réflexif qui, présent au carrefour des civilisations, n'est pas pour autant fortuit, dénué de subjectivité culturelle. Le rire grec, certes exploité au niveau de la polémique sociale ou d'un humour spontané tel qu'il remonte aux temps les plus anciens, ce phénomène émotif qui recompose, comme en Orient, humanité et animalité, a notamment été articulé dans le Dionysisme. Cette réappropriation culturelle du rire à l'époque classique montre que le passage du singe d'une rive à l'autre de la Méditerranée s'est accompagné d'une restructuration de l'imaginaire qu'un détour par le $\mathrm{II}^{\mathrm{e}}$ millénaire minoen nous permet de mieux entrevoir.

Ce coin de la Méditerranée avait sans aucun doute fonctionné comme une charnière cruciale non seulement entre deux civilisations mais également entre le pithecos et un univers religieux qui, d'oriental, est devenu grec. Les fresques de Théra ou celles de $\mathrm{Cnossos}^{28}$ suivaient encore les normes figuratives égyptiennes, touchantes représentations de singes musiciens ou de couleur bleue. Point de corps approximatif dans l'art minoen comme en Grèce archaïque et classique: le trait est précis, l'espèce simiesque très reconnaissable (cercopithèque ${ }^{29}$ ) sans évacuer pour autant un imaginaire qu'on lit en filigrane dans l'activité musicale des primates. Le singe harpiste égyptien vient forcément à l'esprit ${ }^{30}$. Sauf que ces primates sont aussi dotés d'une activité religieuse spécifiquement minoenne: l'animal est représenté devant une divinité féminine dans un rôle d'orant... Il a pu servir d'intermédiaire entre cette déesse protohistorique et les hommes ${ }^{31}$.

(1823) 1992 ou de Gustave Jéquier, Considérations sur les religions égyptiennes, Neuchâtel, 1946.

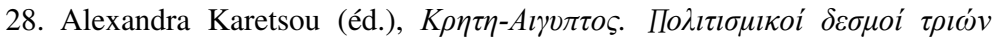
$\chi \imath \lambda l \varepsilon \tau \iota \omega ́ v$, Athènes, 2000 (2 vols.), respectivement fig. 6 et 293.

29. Je remercie M. Eric Delson, anthropologue de l'Université de New York, de m'avoir éclairée à ce sujet.

30. Motifqui apparaît au Moyen-Empire sur des statuettes : voir Henry G. Fischer, "A Scribe of the army in a Saqqarah Mastaba of the Early fifth dynasty», Journal of Near Eastern Studies, 18(4), 1959, p. 233-272.

31. Filippo Carinci, «Scimmie egee», in: E. Cingano et alii (dir.), op. cit. (note 8), p. 95-96 (pl. III, b) et R. Porter, «The Flora of the Theran Wall Paintings : Living Plants and Motifs - Sea Lily, Crocus, Iris and Ivy», in: Susan E. 
Quand les Minoens, donc, s'accommodaient d'imaginaire sans ôter un réalisme biologique précis à l'orientale, la culture grecque du I ${ }^{\mathrm{er}}$ millénaire ancrait ses représentations mentales dans le flou des formes humanoïdes, précisément dans cette hybridité particulière qui s'est en même temps approprié le rire à l'époque classique. La corporéité simiesque grecque doit être éclairée à la lumière d'une histoire longue où elle s'apprécie, dès lors, non pas en termes d'histoire de l'art - de médiocrité des figurations - mais également d'anthropologie religieuse: la question se pose ainsi de savoir dans quelle mesure l'hybridité simiesque relève d'une mise en forme à la fois culturellement originale et accoudée à un précédent religieux bien grec. C'est poser l'hypothèse d'une forme corporelle signifiante du pithecos.

Un autre affranchissement de la réflexion vis-à-vis des habitudes intellectuelles jusqu'ici contractées par les hellénistes consiste à extraire le singe de l'humour - qui prend sens dans l'espace social - pour l'intégrer dans le rire - qui dépasse largement ce cadre caricatural. Or, c'est précisément dans le rire que semble se trouver la pierre angulaire, dans la culture grecque classique, d'une appartenance commune du singe à la sphère sociale comme à la religieuse.

\section{IMAGINAIRE ET RELIGION: LA CONNEXION ENTRE CROYANCES ET RIRE}

À ce stade de l'analyse, l'anthropologue se trouve aux prises avec deux paramètres larges de la pensée antique: l'approche grecque du rire et celle de l'animal. Le rire grec avait une dimension introspective voire cathartique qui n'a pas échappé aux anciens philosophes ${ }^{32}$; et il est intéressant de rappeler que cette forme d'autoréflexion - ou d'autodérision - accompagne, sous des formes variées, les plus anciens systèmes de pensée orientaux. Aussi le singe motive-t-il un rire pleinement anthropologique, universel, et

Sherratt (éd), The Wall Paintings of Thera. Proceedings of the First International Symposium, 30 August - 4 September 1997, Athènes, 2000, p. 620-621.

32. Voir l'usage philosophique du rire chez Socrate selon MarieLaurence Desclos, «Le renard dit au lion... » (Alcibiade majeur, $123 \mathrm{~A}$ ) ou Socrate à la manière d'Esope», in: B. Cassin et alii (dir.), op. cit. note 4, p. 395-422 (notamment p. 405-407). 
suscite dans le regard que l'homme jette sur lui une spontanéité pleine de profondeur. Or, l'approche grecque de l'animal s'insère justement dans une antique réflexion anthropocentrique qui recourt à l'animal, organise à partir de celui-ci une définition de l'homme. L'idée d'humain est marquée depuis l'Orient par un relativisme certain $^{33}$, un type d'anthropocentrisme qui disparaîtra entièrement de la pensée chrétienne (nous y reviendrons). Est-il alors étonnant de trouver le pithecos à la croisée de ces deux paramètres ? Car il est à la fois associé à un rire normatif et à des croyances de régression, de passage ou d'initiation qui interrogent la place de l'homme dans le cosmos.

La présence, en particulier, du singe dans l'univers religieux thébain et sa parenté figurative avec l'artisanat du Cabirion n'apparaît pas surprenante, surtout à une époque - le classique finissant - où le rire est pratiqué aussi bien au théâtre que dans les sanctuaires. Associé à l'animalité dont il explicite la dimension transgressive de l'homme, le rire atteint au $\mathrm{IV}^{\mathrm{e}} \mathrm{s}$. av. J.-C. un degré de prégnance qui n'a d'égal que son efficacité, qu'elle soit sociale ou religieuse $^{34}$. La fréquentation par le singe de l'Ancienne et Moyenne Comédie nous en dit plus : bouffon et goinfre, trompeur et artisan de l'illusion, il a toutes les qualités pour apparaître au theatron au sens grec du terme, c'est-à-dire dans le cadre d'une mise en scène $e^{35}$ où le public se fait le spectateur de sa propre condition. Dans le jeu de

33. Oliver Hellmann, "Antike Berichte über "Symbiose", Kooperation und Interaktion zwischen Menschen und Wildtieren», in: Annetta Alexandridis, Markus Wild et Lorenz Winkler-Horaček (dir.), Mensch und Tier in der Antike. Grenzziehung und Grenzüberschreitung. Symposion vom 7. Bis 9. April 2005 in Rostock, Wiesbaden, 2008, p. 183-203 montre comment les textes grecs traduisent un anthropocentrisme diffus: ce n'est jamais l'homme qui s'adapte au monde animal mais les animaux, mêmes sauvages, qui s'adaptent à l'homme: l'humanisation est donc centrifuge, de l'homme à l'animal, mais ce dernier demeure contigu à l'humain voire intimement lié à lui.

34. Stephen Halliwell, «The uses of laughter in Greek culture», Classical Quarterly, 41, 1991, p. 279-296.

35. Malika Hammou, «Y a-t-il une réflexion sur la mise en scène dans les comédies d'Aristophane?», in: Christophe Cusset, Jean-Claude Carrière, MarieHélène Garelli-François et al. (dir.), Où courir? Organisation et symbolique de l'espace dans la comédie antique. Colloque international, 20-21-22 janvier 2000 du CRATA (Culture, représentations, Archéologie et Théâtres antiques), Toulouse, 2001 (Pallas, 54), p. 291-302: dans la mise en scène des pièces comiques, les gradins ne doivent pas être pensés en tant qu'œuvre architecturale mais en tant que présence du public: «il s'agit (...) de désigner le public en tant que regard (...). De fait, theatron ne signifie jamais que "ce qui regarde"» (p. 295). 
la performance s'effectue ainsi une distanciation du spectateur par rapport à lui-même, l'acteur étant pure transfiguration caricaturale du public qui par ce jeu de miroir parvient ainsi à se situer dans le monde de la cité. Quant au rire religieux de l'époque classique, c'est surtout celui, libérateur, de Déméter et de Dionysos: l'intervention du singe dans ce paysage religieux ne s'expliquerait-elle pas, décidément, par une forme de réflexion sur la situation de l'homme dans le cosmos?

Entre se situer dans le monde de la cité et se situer dans le monde tout court, il n'y a qu'un pas que le singe, acteur bouffon par excellence, franchit aisément. Artisan privilégié du rire dont il serait vain, en Grèce, d'isoler le plan profane de celui du sacré36, le singe se situe au carrefour idéal de deux mondes, celui de la cité (théâtre) comme celui du sanctuaire: son association à la Malophoros de Sélinonte est pour le moins claire à ce titre. L'animal est-il connecté au rire rituel, obscène et effréné, éclatant et sans retenue ${ }^{37}$ ? Pour une figurine comme celle de l'Ashmolean Museum, on ne saurait la relier avec certitude à Dionysos ni à Déméter. En plus d'une ignorance du contexte, l'articulation plausible du pithecos dans l'un ou l'autre univers ne l'associe pas non plus au même type de rire ni aux mêmes croyances.

En effet, le rire dionysiaque est ordinairement décrit comme marqué par l'excès, par une sexualité désinhibée, par un total abandon de soi et une mise en scène de type processionnelle ou pseudo-théâtrale qui normalisent le rire en une «licence performative $»^{38}$. Le rire de Déméter est davantage marqué par la

36. Gregory Nagy, «Introduction and Discussion», in : Eric Csapo et Margaret C. Miller (éd.), The Origins of theater in Ancient Greece and beyond. From Ritual to Drama, Cambridge, 2007, p. 121-125: Aristote fut le premier à scinder le théâtre du rite religieux, la tragédie étant pour lui un art distinct du rite, mais c'est une vision faussée de la réalité grecque (p. 125). Guy M. Hedreen, «Myths of ritual in Athenian vase-paintings of Silens », ibid., p. 150-195, montre que l'imagerie des chœurs présente la trace d'une étroite articulation entre mythe, rite et théâtre.

37. Se trouve-t-on en présence d'une forme de rire fantastique? Le singe parait prioritairement connecté à ce rire intemporel et anthropologique qui traverse les civilisations : sur le rire fantastique moderne, voir la belle synthèse de Denis Mellier, «Mort de rire», in: Denis Mellier et Luc Ruiz (éd.), Le rire fantastique : grotesque, pastiches, parodies (Otrante, 15, 2004), p. 7-12, notamment à propos de l'effet de distanciation et de réflexivité du rire fantastique, associé au grotesque et à l'iconographie (p. 9 et 11).

38. S. Halliwell, Greek Laughter. A Study of Cultural Psychology from Homer to Early Christianity, Cambridge, 2008, p. 181. 
célébration du retour à la vie et par un sens éclatant du gelan, ce rire d'origine divine qui monte et inonde de joie le monde, célébré dans sa capacité de fertilité3 ${ }^{39}$. Bien qu'il soit impossible de proposer une interprétation précise pour chacune des figurines, il est cependant notable d'affirmer que, dans l'ensemble, nos sujets de terre cuite font converger le singe vers des représentations apparentées: Dionysos comme Déméter s'associent à l'aischrologie, aux railleries indécentes et au symbolisme sexuel ${ }^{40}$. D'ailleurs, le singe de l'Ashmolean (illustration 5) était pourvu d'un sexe masculin en érection, à l'instar des satyres et grotesques de la série béotienne et éginète. En outre, Dionysos, à défaut d'avoir été sûrement inséré dans des rites de fertilitée ${ }^{41}$, pourrait bien rejoindre le monde de Cybèle via le schème initiatique.

La figurine du nain de Mélos (illustration 2), mise en relation avec Cybèle et les initiations sexuelles masculines, motive tout particulièrement ce lien que Stephen Halliwell avait laissé ouvert avec un point d'interrogation ${ }^{42}$. La présence d'une créature à connotation dionysiaque comme le singe pourrait connecter Dionysos à l'univers des Corybantes dont on sait que l'initiation se faisait sous la forme de danses hautes en couleurs, sujettes à un ridicule rituellement normé. L'insertion du singe dans ce genre de croyances étonne d'autant moins que le ridicule le caractérise de bout en bout dans la littérature comique. Et sa gestuelle naturelle, mimésis de l'homme qu'il reproduit à l'excès ${ }^{43}$, l'associe également à l'idée d'une danse. N'est-il pas l'animal danseur par excellence chez Lucien, pour qui l'initiation ne va pas sans la danse $\mathrm{e}^{44}$ ?

39. S. Halliwell, ibid., p. 163sq. ou Antonio Lopez Eire, «À propos des mots pour exprimer l'idée de «rire» en grec ancien», in: M.-L. Desclos (dir.), Le rire des Grecs. Anthropologie du rire en Grèce ancienne, Grenoble, 2000, p. 13-43 (notamment p. 20).

40. Voir les sources citées par S. Halliwell, ibid., p. 178-179, notamment celles qui concernent des cultes non-athéniens.

41. Voir les doutes de S. Halliwell, ibid., à propos de la présence de Dionysos aux Haloa d'Athènes (chapitre 4).

42. Ibid., p. 192: Dionysos peut-il être inséré dans des rites initiatiques corybantiques?

43. Jacques Dumont, Les animaux dans l'antiquité grecque, Paris, 2001, p. 112.

44. Dans Le pêcheur, 36, les singes sont danseurs professionnels à la cour de Ptolémée alors que dans La danse, 15, Lucien écrit qu'il n'y a pas un seul culte à mystère ignorant la danse. Peut-on appliquer cette affirmation à la Grèce archaïque ou classique? 
Dans l'art laconien archaïque, n'est-il pas représenté avec des cômastes ${ }^{45}$ ?

L'imagerie simiesque apparaît incontestablement plus riche qu'on pourrait le penser, comme sans doute les textes, reliques possiblement résiduelles de représentations religieuses que les chercheurs ont jusqu'à présent considérés au plan de l'humour social. Du point de vue du pithecos et de sa double participation aux mondes de Dionysos et de Déméter, les distinctions franches en matière de rires rituel et social s'estompent. De cet angle d'approche, le singe nous en apprend déjà beaucoup qui intéresse la présente étude: il célèbre diverses formes de marginalités, initiatiques, comportementales... celles-là même qui, sous la forme du rire, l'introduisent dans un univers religieux où l'écart se fait norme. L'animal semble s'ériger en véritable topos du rire rituel, à l'interface sans doute de diverses formes de rires, comme il se trouve à l'interface de la comédie et de la religion, surtout à l'époque classique. Incarnation de pathos dans ce monde de sophrosynè, le singe paraît inséparable de l'émotion que le theatron comme le gelan cultivent communément. Finalement, le singe ne serait-il pas le médiateur idéal entre l'homme et ce qui le dépasse?

\section{DES HOMMES REPRÉSENTÉS COMME DES SINGES?}

Pour en revenir à nos petites figurines béotiennes, l'hypothèse d'une représentation sous la forme animale de dédicants renvoie à des croyances religieuses connues pour leur caractère local et leur ancienneté, conceptions archaïques de dieux et de l'homme

45. Illustration 4. La danse connecte-elle aussi directement le singe à l'idée d'effémination, ou du moins d'absence de virilité ? Cette dernière idée affleure par exemple dans les Acharniens (v. 117-122) où le singe est l'animal imberbe, soumis à un partenaire viril. Dans ce passage de la pièce d'Aristophane, les citoyens Clisthène et Straton, traités de «singes », font fonction d'eunuques du Grand roi. Selon Orfanos Charalampos, Les sauvageons d'Athènes ou la didactique du rire chez Aristophane, Paris, 2006, p. 54-61, l'allusion à l'animal s'explique par sa ruse. Or, cette allusion n'évoque-t-elle pas la transgression sexuelle visible ailleurs, comme sur la figurine de Mélos? Il me semble que les deux citoyens sont autant traités de vils flatteurs que de "moins homme", soumis à l'autorité virile des Perses dont ils se sont fait les esclaves. 
usant de zoomorphisme ${ }^{46}$. Réflexion religieuse sur la forme, les figurines simiesques de Grèce centrale nous rappellent de loin en loin un type d'images d'Arcadie: celui des créatures à tête animale exhumées dans le sanctuaire de la Despoina de Lycosoura ${ }^{47}$. En plus de renvoyer à certains aspects locaux de divinités, ces images fabriquent également des représentations de l'homme inséparables de l'animal. Dans ce genre d'anciens systèmes de pensée, l'animal s'érige en outil conceptuel ${ }^{48}$, quitte à relativiser sa propre nature. Ainsi, la proximité du singe d'avec le satyre laisse augurer un rôle ou une médiation assez précise du pithecos dans la religion béotienne classique: celui de visualiser, de mettre en forme - au sens premier terme - l'Autre caché dans l'homme, à l'écart de ses expériences ordinaires. Certaines figurines en terre cuite constitueraient donc bien une construction iconographique signifiante par rapport à une expérience religieuse. La difformité qu'elles mettent en exergue est susceptible d'évoluer, semble-t-il, dans un registre émotionnel.

Le choix spécifique du singe évoque du point de vue de l'anthropologie religieuse un type de croyance déjà observable en Égypte: le singe peut occasionnellement passer pour être une manifestation du divin invisible dans le visible ${ }^{49}$. Évidemment, on ne saurait appliquer stricto sensu cette logique à la culture grecque d'autant que le singe, en Égypte, partage cette fonction avec bien d'autres animaux. Pourtant, on pourrait se trouver en présence des mêmes conceptualisations du monde d'un point de vue grec, celui

46. Pour Liliane Bodson, 'IEPA Z $\Omega I A$. Contribution à l'étude de la place de l'animal dans la religion grecque ancienne, Bruxelles, 1978, l'association de l'animal à la divinité découle de conceptions archaïques jugées insurpassables pour accéder au divin, vivaces notamment en Arcadie et en Béotie. Cet imaginaire fonctionne dans le giron de certaines divinités particulières, comme Artémis et Dionysos (p. 165).

47. Voir l'interprétation de ce matériel par Emma Aston, «Hybrid Cult Images in Ancient Greece: Animal, Human, God», in: A. Alexandridis et alii (dir.), op. cit. (note 33), p. 481-502 qui associe ce zoomorphisme à la Déméter de Phigalie et suppose une fonction de l'hybridité dans le culte. À noter aussi la découverte de figurines en terre cuite de femmes à tête animale à Lykosoura (époque romaine), images vraisemblablement votives (p. 488sq.).

48. Claude Lévi-Strauss, La pensée sauvage, Paris, 2008, chapitre v ou VII, a bien sûr analysé les raisons et le fonctionnement de la «naturalisation» (ou animalisation) de l'homme: le recours à l'animal constitue «une classification «à pas variable», donnant au groupe qui l'adopte, sans changer d'instrument intellectuel, le moyen de se mettre «au point» sur tous les plans, du plus abstrait au plus concret, et du plus culturel au plus naturel» (p. 700).

49. Voir infra note 60 (quand je cite Aufrère). 
qui réfléchit à l'homme en présence du divin, qui réfléchit à la forme prise par l'un et par l'autre et qui use de l'animal pour ressentir l'Autre dans l'homme, un autre immédiat, transparent au monde, médiateur de l'invisible ${ }^{50}$. Pour l'homme qui cherche à se libérer de lui-même, le dionysisme permet de se découvrir comme seuil, d'ouvrir à un rire mystique et théurgique et à une expérience du

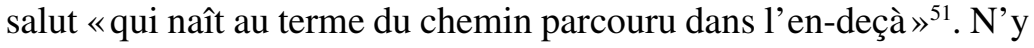
aurait-il pas dans le singe quelque chose du trickster au sens où le définissait Laura Lévi Makarius ${ }^{52}$ ? L'image d'un être sacré sous des aspects misérables et dégoûtants, bouffon et phallique, médiateur donc, chargé d'exécuter la transgression nécessaire à une efficacité de type mystique?

Quand, dans le judaïsme antique, l'animal a accès au Créateur en étant en même temps relativement distancé de l'homme ${ }^{53}$, en Grèce, les croyances réduisent particulièrement cet écart en usant de l'animal pour expliciter l'Autre caché au plus profond de chaque être humain, cet Autre en soi que, dans ses transes ou son ivresse, le dionysisme sait révéler ${ }^{54}$. De fait, les petites figurines béotiennes qui montrent occasionnellement des «singes» avec un objet dans les mains sont susceptibles de formaliser une image de l'humain à la lisière de sa nature. Ces terres cuites «fixent» peut-être dans l'image une action religieuse (offrande ?) mais, dans tous les cas, la créature représentée se trouve momentanément distancée d'elle-même:

50. Ce type de vision du monde est observable dans les cultures où un lien affectif lie l'homme à l'animal et où l'animal-médiateur entre visible et invisible sert à classer, soit sur le mode totémique soit sur le mode métaphorique: voir les remarques de Philippe Borgeaud, «L'animal comme opérateur symbolique», in: P. Borgeaud et Yves Christe (éd.), L'animal, l'homme, le dieu dans le ProcheOrient ancien. Actes du colloque de Cartigny 1981, Louvain, 1984 (Cahiers du Cepoa, 2), p. 13-20.

51. Jean Brun, Le retour de Dionysos, Paris, 1976, p. 244-246.

52. Le sacré et la violation des interdits, Paris, 1974, chapitres V et VI.

53. Albert De Pury, «Animalité de l'homme et humanité de l'animal dans la pensée israélite. Comment l'homme se définit-il par rapport à l'animal?», in: P. Borgeaud et Y. Christe (éd.), op. cit. (note 50), p. 47-70.

54. Voirl'étude deF.Lissarague, «Satyres, sérieux s'abstenir», in:M.-L.Desclos (dir.), op. cit. (note 39), p. 109-119, à propos des satyres sur les vases à boire qui révèlent par l'inversion des bons usages du symposion l'Autre caché en chaque convive (p. 118). Pour l'hypothèse du «satyre-trickster», héros bouffon faisant passer la création par le chaos, voir Bernard Sarrazin, «Rire et sacré, de Dionysos à Nietzsche, du christianisme à l'islam », in : Dominique Bertrand et Véronique GélyGhédira (éd.), Rire des dieux, Clermont-Ferrand, 2000, p. 407-417. 
est-ce le renvoi à une expérience religieuse émotionnellement vécue, exprimée dans le rire?

La dilatation des formes corporelles humaines que le singe met en œuvre, comme les grotesques, ne serait-elle pas une explicitation visuelle d'une mise en relation intime avec la divinité ? Se trouverait-on en présence d'un type de métamorphose, médiatrice obligée de la rencontre de l'homme avec l'indicible... une médiation qu'une figure humanoïde et intermédiaire comme le singe pourrait, au mieux, traduire? Serait-on en présence d'un type d'expérience du passage ? La question doit être posée car le passage pourrait donner un sens ultime à l'animalisation de l'homme, et le singe, mimésis si troublante, pourrait peut-être bien n'être d'abord que cela dans l'univers dionysiaque: un homme déformé, interprète idéal d'une religiosité transgressive du type de celle palpable sur la figurine archaïque de Mélos $^{55}$. La difformité simiesque pourrait bien constituer un type d'hybridation en soi, celle de l'homme-animal qui s'ancre dans l'expérience d'une altérité pulsionnelle en marge de l'humanité socialement affichée ${ }^{56}$. Dans des croyances religieuses où l'être social se trouve soudainement marginalisé, l'animal semble apparaître comme moyen symbolique, comme référent culturel de distanciation de l'homme à l'homme.

55. Sur les danseurs dionysiaques qui évoquent un moment spécifique de la vie de l'homme pendant lequel ce dernier éprouve l'impression d'être incomplet, sur le Dionysisme célébré pendant les périodes transitionnelles de la sexualité masculine, voir Cornelia Isler-Kerényi, Dionysos in Archaic Greece. An Understanding through Images, Leyde, Boston, 2007, p. 59sq. et p. 232sq. On peut également se poser la question de la ressemblance entre le singe dionysiaque et la figure du «danseur rembourré» dont Marie-Hélène Delavaud-Roux, Les danses dionysiaques en Grèce antique, Aix-en-Provence, 1995, a relevé la spécificité béotienne: contrairement aux autres régions, les danseurs béotiens sont plus caricaturés et leurs proportions anatomiques peu respectées: voir l'excellent exemple d'une pyxide béotienne de $-575 \mathrm{ca}$. (p. 67-68) montrant des danseurs à la gestuelle exubérante et aux bras allongés à l'extrême, des détails qui ne sont pas sans rappeler le corps du singe.

56. On se trouverait en présence de ce que Gilbert Durand, Les structures anthropologiques de l'imaginaire, Paris, 1992, p. 38, appelle la «genèse réciproque », c'est-à-dire l'échange perpétuel dans l'imaginaire entre les pulsions subjectives et les intimations objectives du milieu social. Sur la traduction d'affects à partir des références culturelles d'une société, par exemple mythologiques, et sur le désir humain de variation du corps, voir l'analyse anthropologique de Pierre Ancet, «Brève histoire des idées et représentations des monstres hybrides entre l'homme et l'animal», in: Valérie Camos, Frank Cézilly, Pierre Guenancia et alii (dir.), Homme et animal, la question des frontières, Paris, 2009 (collection «Update Sciences et Technologies»), p. 43-54. 


\section{HYPOTHÈSES}

Si le singe a traversé de part en part la sphère du ridicule et du comique dans la culture grecque, notamment à l'époque classique, il n'est pas interdit de penser qu'un usage spécifique et religieux ait fonctionné vers la même époque. Que le singe ait pu caricaturer le savant ou le musicien en Grèce, on ne le niera pas: l'animal était dressé pour amuser son public comme en témoignent à la fois la littérature et l'iconographie ${ }^{57}$. Mais certaines figurations pourraient broder un sens supplémentaire qui n'est pas anodin, encore moins «profane».

Le singe semble donc avoir fonctionné bien au-delà de sa nature prosaïque de bestiaire exotique. Certaines images exploitent l'animal pour traduire un écart porteur de sens. Le pithecos, en tant que forme relativement proche de l'homme mais simultanément distancé de la physiologie de ce dernier, a pu particulièrement servir cette préoccupation-là: rendre compte d'un type d'expérience religieuse qui est altérité ou «confin», ancrée dans la confrontation de l'homme avec une autre dimension de lui-même; une dimension qui ne lui est pas totalement étrangère mais qui, le dépassant pourtant, appartient à l'univers des dieux. Le singe a tout l'air de participer à une perception religieuse du monde dans laquelle l'exotisme appuie une introspection ou sert à la circonscrire comme type d'altérités ${ }^{58}$. Créant un écart, le pithecos est capable d'organiser une mise en perspective entre natures humaine et animale. Il suscite tantôt un décalage, tantôt un ajustement à l'homme, créature surprenante dont l'effet pourrait être actif sur le plan de divers degrés d'émotion: du rire comique à d'autres moins anodins et peut-être plus cathartiques, dépositaires d'une vérité sacrée ${ }^{59}$. Aussi faut-il sérieusement retenir

57. Pour les textes, on retiendra notamment Lucien, Le Pêcheur, 36. Quant aux images, voir supra note 5 auxquels s'ajoutent bien d'autres exemples: singe accomplissant un tour (R.A. Higgins, op. cit. [note 5], $\mathrm{n}^{\circ}$ 796), singes sur une mule (F. Winter, op. cit. [note 5], n ${ }^{\circ} 223,1$ et 1 b), etc.

58. Ce que l'on peut affirmer à la suite des conclusions de L. Bodson, «Les animaux dans l'antiquité: un gisement fécond pour l'histoire des connaissances naturalistes et des contextes culturels », in : Christian Cannuyer, Denyse FredericqHomes, Francine Mawet et alii (éds.), L'animal dans les civilisations orientales, Bruxelles, Louvain, 2001 (Acta Orientalia Belgica, 14), p. 1-27.

59. N'en trouve-t-on pas un écho au niveau philosophique ? Rappelons que dans Pindare, Pythique, II, 72-73, le singe sert à dénoncer les illusions dont est victime l'homme qui se laisse aller à la flagornerie, à l'ignorance: il dessille les yeux de l'homme qui se détourne d'une vérité d'ordre moral. 
l'hypothèse, aux tournures anthropologiques, de la relation entre cet animal et un type de métamorphose, un rapport qui se pose de l'Égypte à la Grèce ${ }^{60}$. Le singe comme animal pourrait apporter une contribution importante à la question de la définition du genre humain telle qu'elle commence à être abordée dans les recherches concernant le corps ${ }^{61}$. La Grèce, si elle est placée sur le cours d'une créativité humaine plus antique encore qu'elle, et si elle est pour partie le berceau de notre sensibilité vis-à-vis du singe, a bien élaboré au travers de cet animal des motifs iconographiques et philosophiques propres qu'une ultime comparaison avec le regard chrétien permet, en dernière instance, de bien évaluer.

\section{Des GReCs À DARWIN}

La pensée religieuse grecque relève d'un anthropocentrisme qui, semble-t-il, sait opérer une distanciation de l'humanité par rapport à elle-même et à partir de l'animal ce dont l'anthropocentrisme chrétien occidental est précisément incapable, notamment depuis le $\mathrm{XIX}^{\mathrm{e}} \mathrm{s}{ }^{62}$. Pour clore cette analyse, recourons à une représentation de Darwin en compagnie d'un singe (illustration 9). Cette caricature vient à point nommé éclairer, comme un négatif, une certaine philosophie grecque de l'être et aussi le fossé entre deux approches du genre humain pourtant exprimées au moyen du même type d'images : l'hybridité homme-animal. Finalement la pensée grecque et la moderne présentent des similarités fallacieuses.

60. Quoique de manière différente: si l'animal apparaît en Égypte comme un médiateur entre mondes humain et divin et s'il met en branle tout un imaginaire de métamorphoses, ce dernier, fondé sur l'observation des comportements animaux, opère des amalgames entre espèces: par exemple, le chat peut apparaitre comme la métamorphose de la lionne quand elle est visualisée dans sa forme apaisée: voir Sydney H. Aufrère, Thot Hermès l'Égyptien. De l'infiniment grand à l'infiniment petit, Paris, 2007 (Collection Kubaba, Série Antiquité, XIII), p. 98sq.

61. A. Alexandridis, M. Wild et L. Winkler-Horaček, "Einleitung", in: A. Alexandridis et alii (dir.), op. cit. (note 33), p. 3-4.

62. Auparavant, les contours de l'homme ne sont pas aussi précis: sur l'évolution historique du rapport homme-animal dans la pensée occidentale, voir Giorgio Agamben, L'ouvert. De l'homme et de l'animal, Paris, Turin, 2002, p. 40sq. et p. 52sq. ou Elisa Romano, «Premessa», in: Fabio Gasti et Elisa Romano (dir.), "Buoni per pensare". Gli animali nel pensiero e nella letteratura dell'antichità. Atti della II Giornata ghisleriana di Filologia classica (Pavia, 18-19 aprile 2002), Pavie, 2003, p. 11. 
L'illustration 9 est la copie d'une caricature parue dans le London Sketch Book à l'occasion de la publication de L'origine des espèces de Darwin en 1859. L'homme grec considérerait sans doute cette image avec un regard amusé mais en rien abasourdi, contrairement au chrétien qui partage avec le monde savant du $\mathrm{XIX}^{\mathfrak{e}} \mathrm{s}$. une approche somme toute commune de l'homme: celle fondée sur le préétabli, qu'il soit religieux ou scientifique. Il y avait également du préétabli chez Darwin quand il pensait son évolution en termes de degrés d'évolution, créant des êtres de développement supérieur à d'autres ${ }^{63}$; et Thomas Huxley, «le bouledogue de Darwin», faisait du langage le propre de l'homme. En 1893, peu après le catéchisme de l'abbé Maudoit, l'encyclique de Léon XIII Providentissimus n'opposait pas davantage le théologien au savant parce que ni l'un ni l'autre ne songeaient à remettre en cause la frontière entre l'animal et l'homme: ne fut-elle pas créée de si longue date par le christianisme et entretenue par l'essor des sciences humaines? Le chrétien n'a pas davantage besoin de recourir à l'animal pour représenter Dieu puisqu'il connait Son visage. C'est tout le contraire du dionysisme où l'enthousiasmos, qui transfigure le fidèle au point de le faire animal ${ }^{64}$, donne au zôon un rôle majeur de dévoilement du divin, visualisé comme son mode d'accès privilégié: nul doute que le singe ait, comme le satyre ${ }^{65}$, prêté son concours à ce genre de croyances.

Par ailleurs, dans le christianisme, l'humain est devenu irréductible à l'animal en raison également d'une autre conception

63. «Les enfants des sauvages manifestent une tendance plus forte à allonger leurs lèvres que les enfants des Européens civilisés, ce qui est analogue à la mimique des jeunes orangs-outangs ou des jeunes chimpanzés »: Charles Darwin, L'expression des émotions chez l'homme et les animaux, Paris, 1874, p. 253, cité par Jacques Arnould, Requiem pour Darwin, Paris, 2009, p. 162.

64. Dans le christianisme, non seulement l'animal ne peut plus représenter Dieu mais il peut même incarner une partie mauvaise de la création pour les manichéens ou les puritains: voir les synthèses de C. Isler-Kerényi, «I misteri di Dioniso», in: Angelo Bottini (dir.), Il rito segreto. Misteri in Grecia e a Roma, Roma, Colosseo, 22 Iuglio 2005/8 Gennaio 2006, 2005, p. 71, et de Jean Prieur, Les animaux sacrés dans l'antiquité. Art et religion du monde méditerranéen, Rennes, 1988, p. 191-192.

65. Dionysos s'empare des gestes et des contours corporels de l'homme au point de le transfigurer en animal: le cas des satyres est évoqué par Françoise FrontisiDucroux, «Images du ménadisme féminin: les vases des 'Lénéennes' », dans L'association dionysiaque dans les sociétés anciennes. Actes de la table ronde organisée par l'École française de Rome (Rome, 24-25 mai 1984), Rome, 1986 (Collection de l'École française de Rome, 89), p. 165-176. 
du salut, celle qui concerne les descendants d'Adam, tous pécheurs mais aussi parcelles de divin: ce présupposé théologique plaçait l'homme, une fois pour toutes, bien au-dessus de l'animal, par éviction ontologique de ce dernier. En Grèce ancienne, le salut qui peut s'insérer dans des rites initiatiques est sans doute d'abord une sorte de promotion au sein des hiérarchies cultuelles et sociales, une expérience qui fabrique une nouvelle personne, à la fois dans la conscience que cette personne a d'elle-même, résultat de ce qu'elle a vécu par ces rites, et dans la conscience que les autres ont de cette personne: le salut s'insère dans une économie politique du statut des personnes ${ }^{66}$, un discours auquel le singe, là encore, a tout l'air de participer: ne s'exhibe-t-il pas comme tel surtout dans le regard des autres - theatron?

Ces différences de culture religieuse ne rangent évidemment pas du tout le singe au niveau des mêmes enjeux théologiques. Le paganisme grec savait absorber l'animal en l'homme sans créer le déclassement ontologique qu'un tel rapprochement crée dans le christianisme. Dans le monothéisme, c'est la dégénérescence de l'homme en animal que le singe incarne, abomination du simia dei, homme dégradé qui chute de sa condition pour refuser le sacrifice du Christ. Aussi cette image de Darwin qui tend un miroir à un singe est-elle particulièrement significative du rapport impossible de l'homme à l' animal dans un monothéisme particulièrement strict en la matière, et acerbe - corrosif - vis-à-vis du savant dont le seul tort est d' avoir osé relever, malgré des préétablis philosophiquement apparentés au christianisme, les ressemblances entre homme et animal. On reconnaît d'abord dans le geste du miroir tendu une allusion à l'iconographie médiévale qui exhibe le singe avec cet ustensile de luxure morale: l'homme pécheur, si loin de Dieu, y reconnait alors sa face hideuse. Contrairement aux créatures hybrides grecques, Darwin n'est donc nullement animalisé sur cette image: il est animal-tout-court, sa grosse tête créant moins une hybridité qu'un jeu visuel destiné à identifier le savant caché dans ce corps poilu. L'animalisation de l'homme ne saurait être qu'effroyable - on songe à l'anecdote amusante de l'épouse de

66. Bruce Lincoln, «The Initiatory Paradigm in Anthropology, Folklore, and History of Religions », et James Redfield, «Initiations and initiatory experience», in: David B. Dodd et Christopher A. Faraone (éd.), Initiation in Ancient Greek Rituals and Narratives, Londres, New York, 2003, p. 241-254 et p. 255-259. 
l'archevêque de Cantorbéry horrifiée par la thèse de Darwin ${ }^{67}$ - ; et, sur le plan de l'image, elle ne saurait être que superficielle, incomplète, apparente, puisque blasphématoire dans son principe.

La pensée chrétienne se refuse à jauger l'homme à partir de l'animal là où, précisément, le paganisme grec voyait l'occasion d'identifier l'Autre qui permet à l'homme d'être encore mieux luimême. Mais le singe était déjà le sceau de Satan dans l'Ancien Testament ${ }^{68}$, une image que le judaïsme a transmise telle quelle au christianisme. Qui plus est, une autre forme de vécu moral et religieux apparut à la fin de l'Antiquité qui fermait l'homme au rire $^{69}$. Et c'était sans compter avec l'apport du stoïcisme ${ }^{70}$ qui avalisait définitivement, jusqu'aujourd'hui, la fermeture de l'homme à l'animal ${ }^{71}$. Point d'entre-deux, point de réification de l'être dans cette vision du monde, et c'est ce qui rend encore si difficile notre propre accessibilité à la figure religieuse du pithecos, tombé dans l'oubli : il est temps de le faire renaître en Dionysos et en Déméter ${ }^{72}$.

ISTA, Université de Franche Comté

32 rue Mégevand

25030 Besançon Cédex

karin.mackowiak@univ-fcomte.fr

67. «Seigneur, faites que cela ne soit pas vrai !... et si, par malheur, c'était vrai, faites au moins que cela ne se sache pas !»: A. De Pury, op. cit. (note 53), p. 50.

68. Par exemple Isaïe, 34, 14. Et le Talmud oppose strictement le singe à Dieu en raison de son apparence physique, notamment ses poils (Masehet SanhedrinHazal). Le singe (kof en hébreu) est du côté du sauvage (désert) donc de la laideur et du diable.

69. Voir notamment les écrits de Grégoire de Nysse et de Jean Chrysostome où le rieur païen apparaît dans toute sa folie, sa vulgarité et sa honte, le rire s'opposant désormais au Christ et à la mortification corporelle (sources citées par S. Halliwell, op. cit. (note 38 ), p. 9 et p. $495 \mathrm{sq}$ ).

70. Gilbert Simondon, Deux leçons sur l'animal et l'homme, Paris, 2004, chapitre 1 .

71. La biologie de l'évolution d'aujourd'hui, continuiste, affirme, malgré un point de vue non anthropocentré, qu'il existe une différence de degrés entre l'homme et les animaux, en prenant pour exemple notamment les grands singes. Ainsi, la distinction entre les deux genres continue à agir, sans remise en question de l'unité biologique substantielle entre tous les êtres: voir le bilan de JeanPierre Sylvestre, «Présentation», in: V. Camos et alii (dir.), op. cit. (note 56), p. 7-8. Pourtant, certains éthologistes commencent à identifier le rire des animaux.

72. J'adresse tous mes remerciements à Hélène Bernier-Farella (Université de Cergy-Pontoise) pour ses précieuses remarques à propos de cet article. 

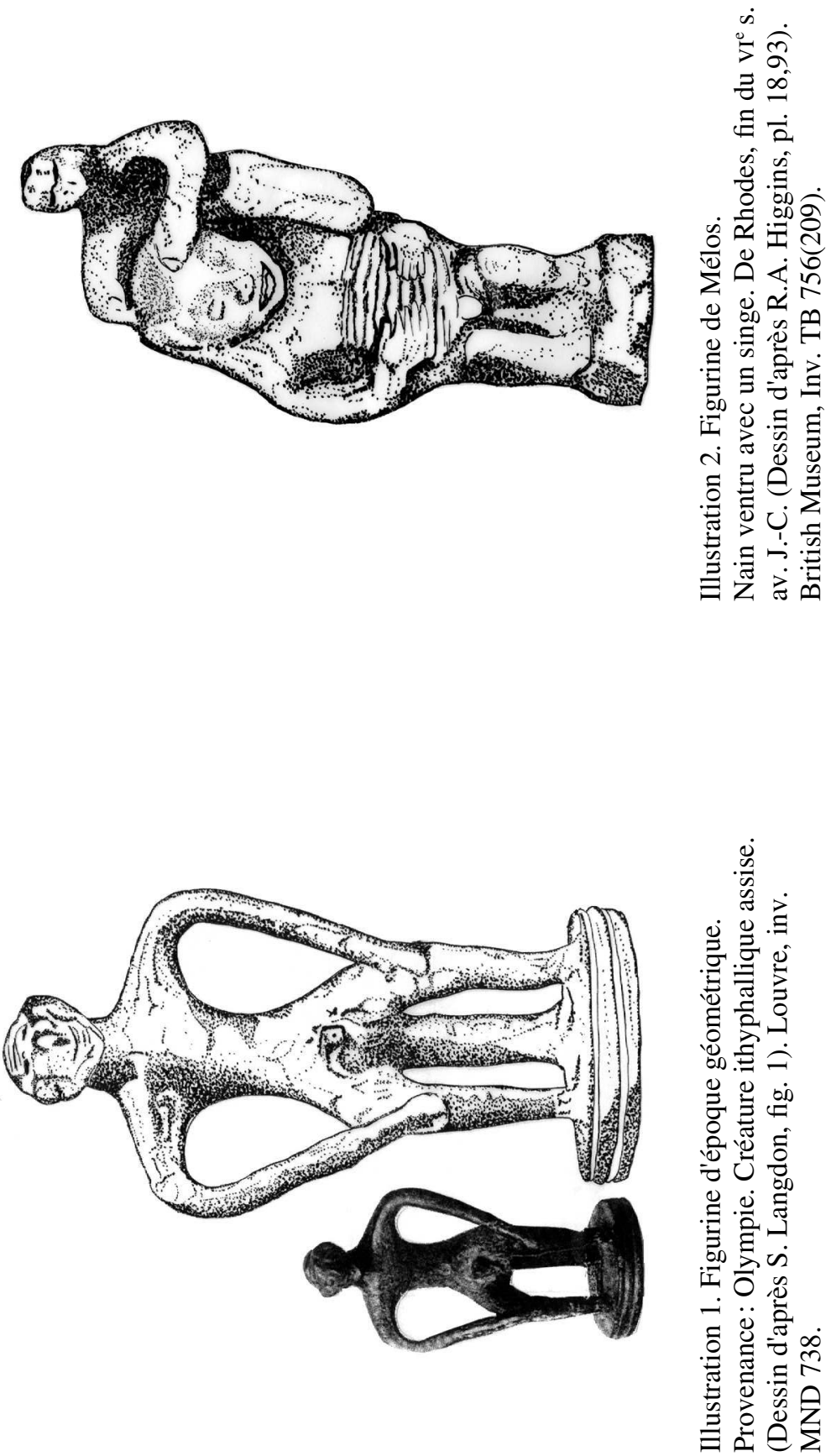

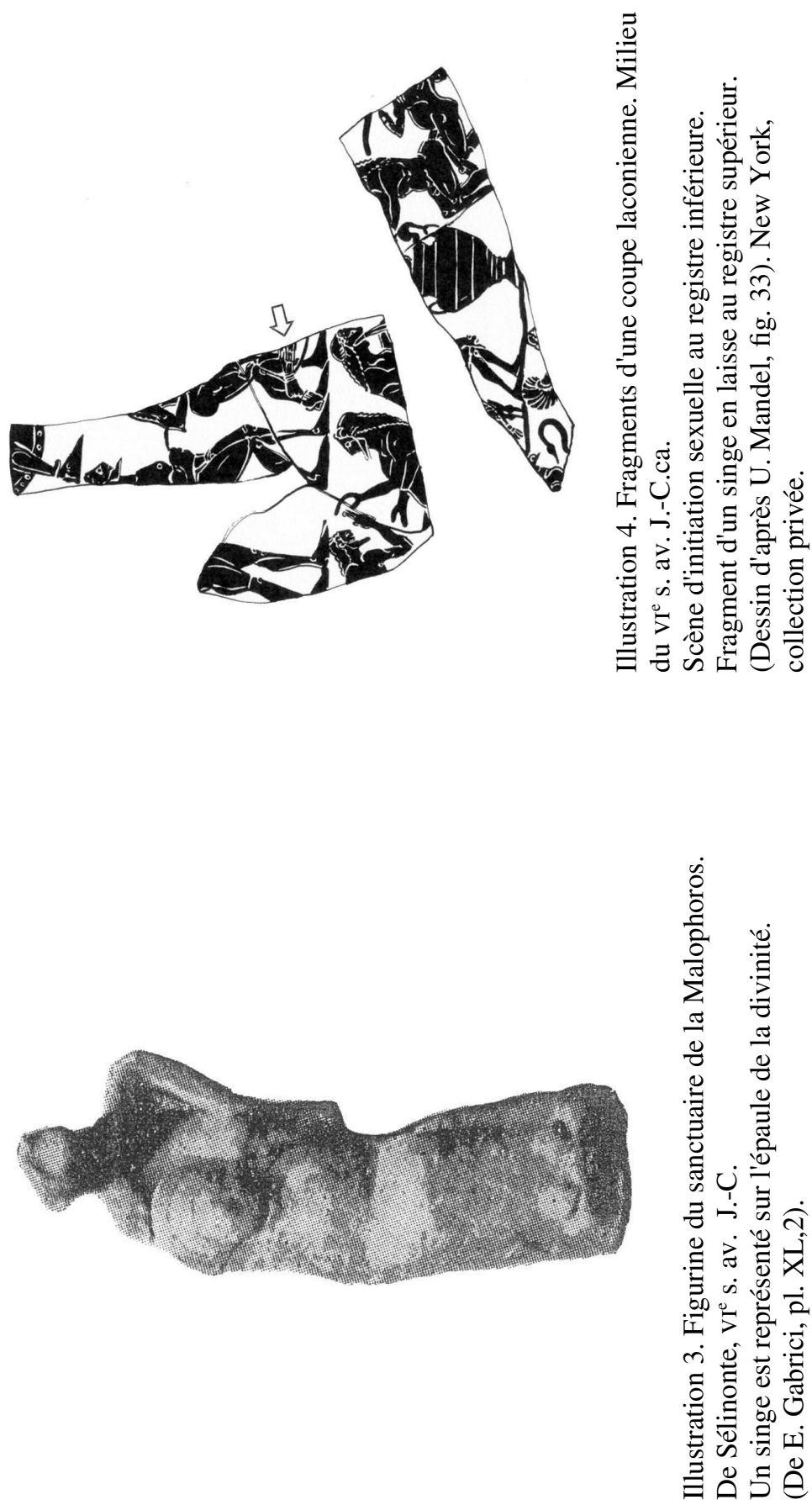

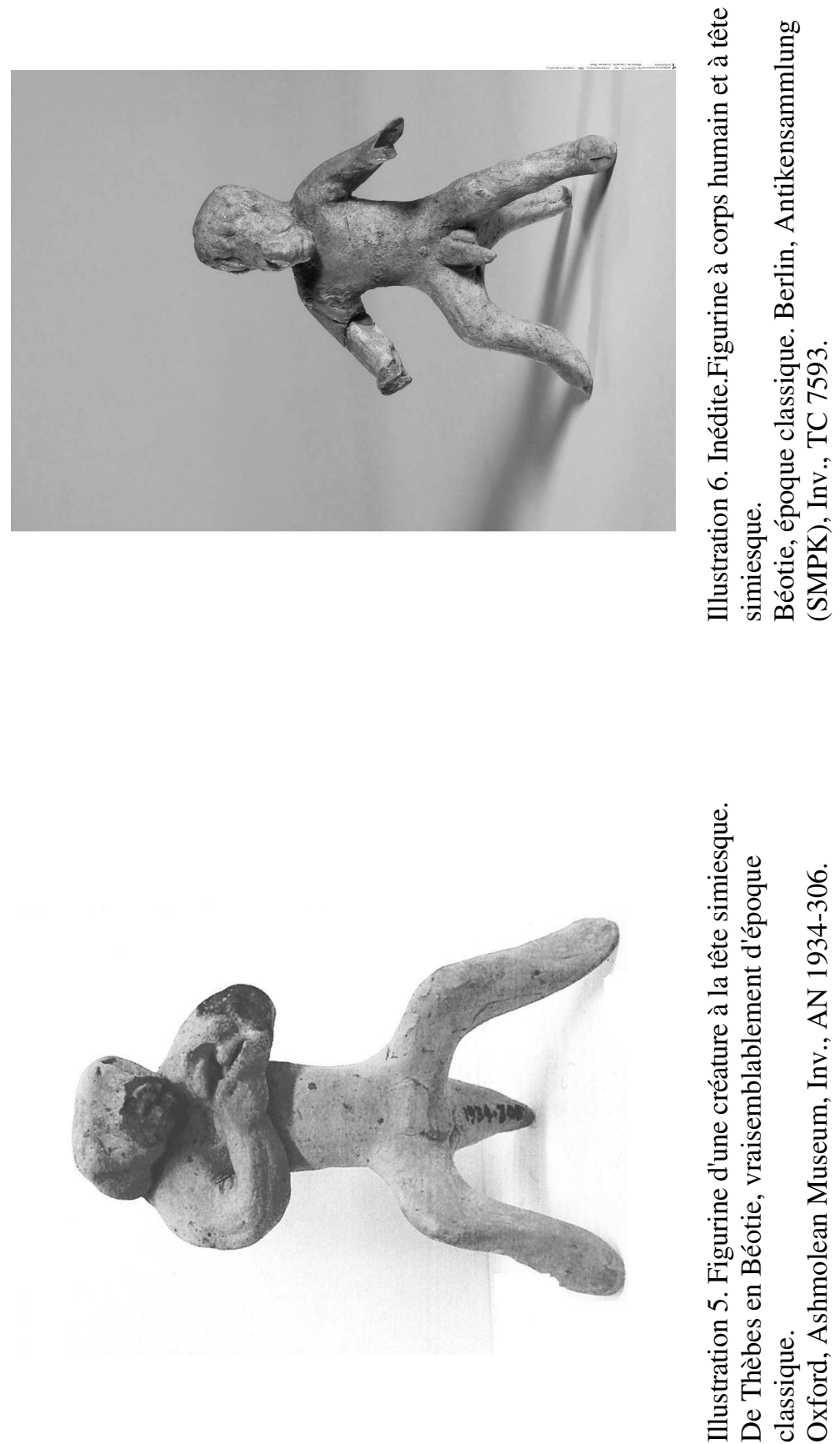


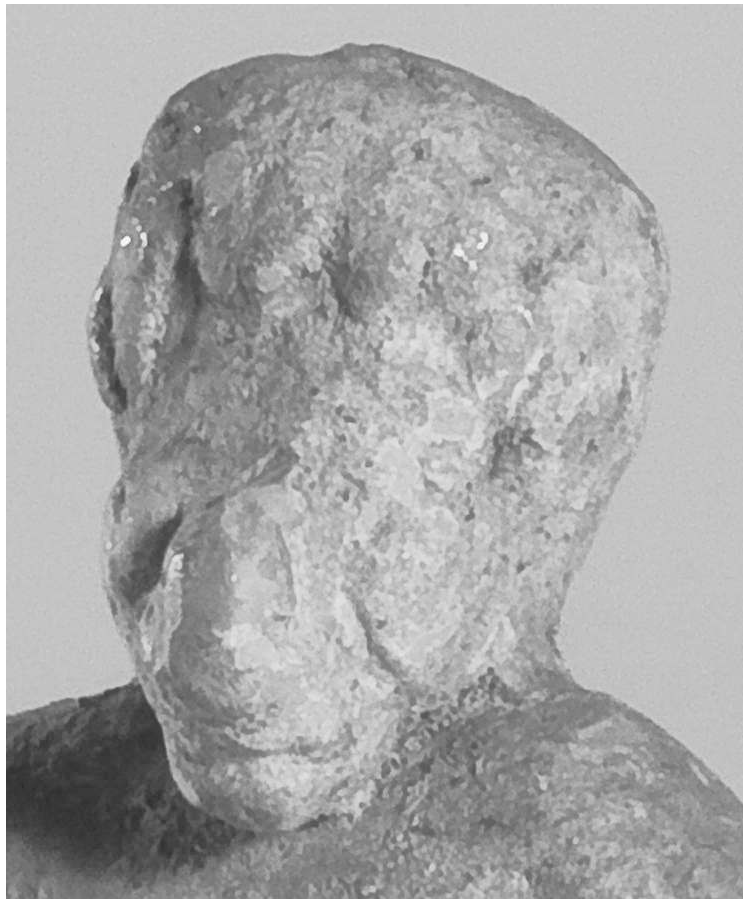

Illustration 7. Gros plan sur la tête de la figurine de Berlin.

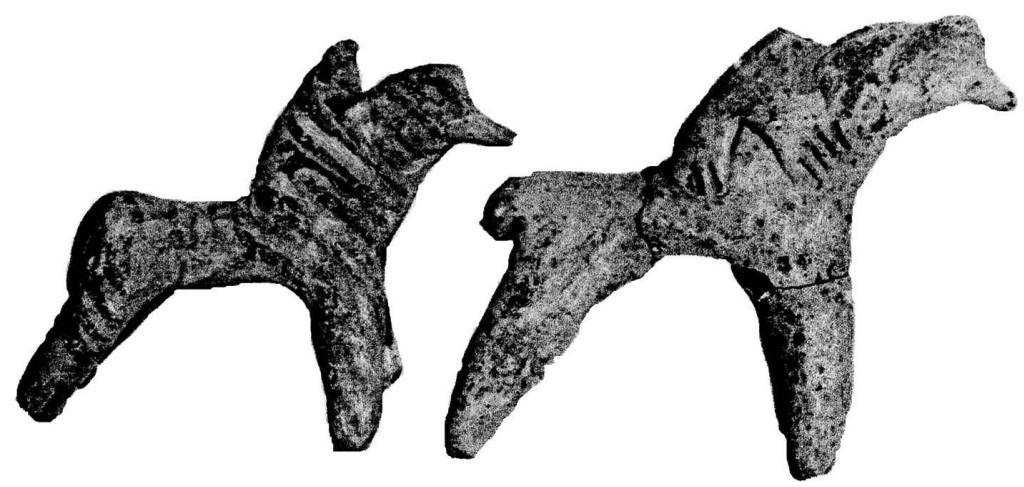

Illustration 8. Créatures identifiées à des singes qui chevauchent une monture. Figurines de Béotie, $\mathrm{VI}^{\mathrm{e}}$ s. av. J.-C. (De P.N. Ure, pl. 15, $\mathrm{n}^{\circ} 145.96$ et 97 ). 


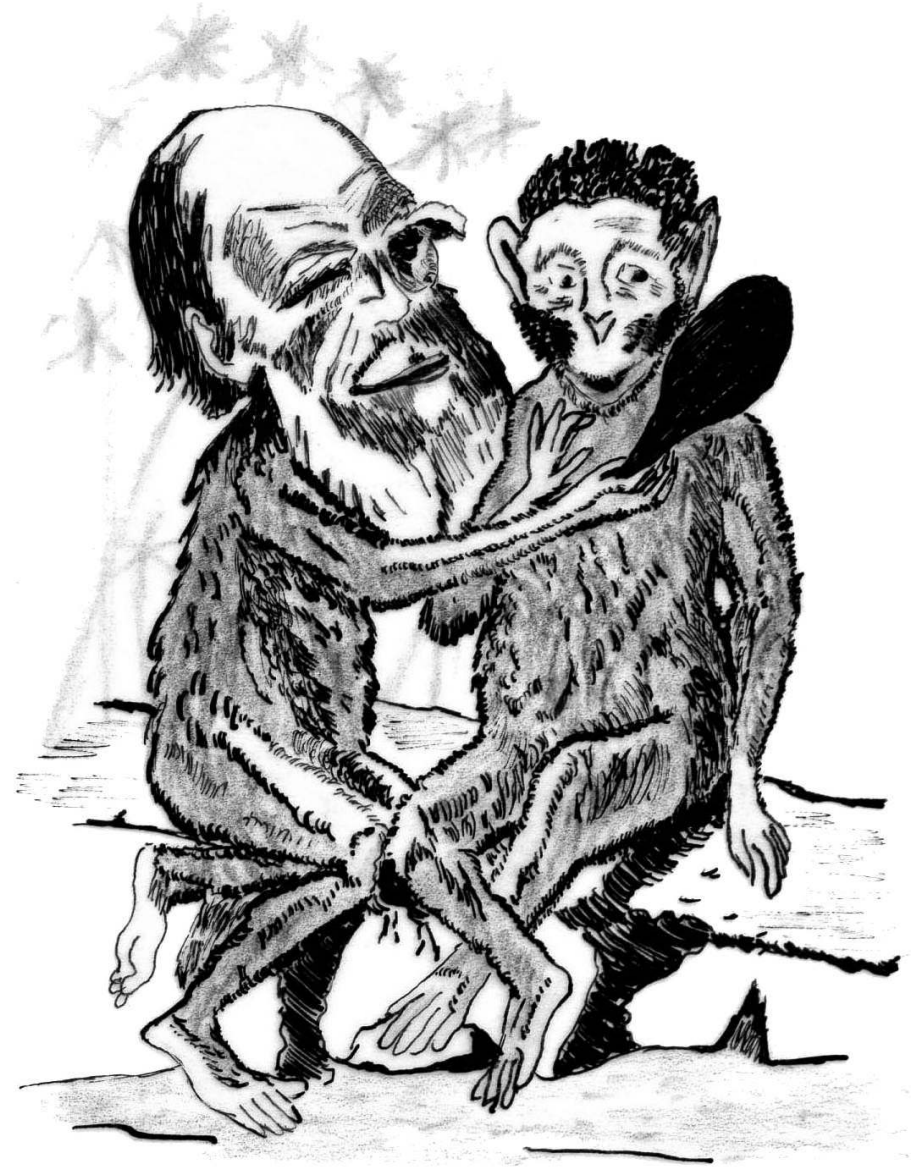

Illustration 9. Caricature de Darwin parue dans le London Sketch Book en 1859. (Dessin d'après Jacques Legrand (éd.), Chronique de l'humanité, Paris, 1986, p. 895). 